\title{
DESIGN AND IN VITRO EVALUATION OF FAST DISSOLVING FILMS CONTAINING HP ß CD INCLUSION COMPLEXES OF LAMOTRIGINE
}

\author{
H Doddayya ${ }^{*}$, Shrishailgouda $S$ Patil ${ }^{1}$, G Ramya Sree ${ }^{1}$, Waseem $\mathbf{H}^{1}$, Udupi RH $^{2}$ \\ ${ }^{1}$ Department of Pharmaceutics, N.E.T Pharmacy College, Navodaya Nagar, Raichur, Karnataka. India \\ ${ }^{2}$ Department of Pharmaceutical Chemistry, N.E.T Pharmacy College, Navodaya Nagar, Raichur, Karnataka, India
}

\begin{abstract}
:
Objectives: The objectives of the present study is to design and evaluate fast dissolving films of lamotrigine using HP $B$ CD inclusion complexes with an aim to achieve rapid drug release thereby controlling the seizures in the shortest possible time.

Design: Inclusion complexes of lamotrigine were prepared with hydroxypropyl $\beta$ cyclodextrin (HP $\beta$ CD) employing different methods to improve the solubility and mask the bitter taste of the drug. Fast dissolving films were prepared by incorporating the developed inclusion complexes and using hydroxypropyl methylcellulose (HPMC) and polyvinyl alcohol (PVA) as film forming agents and other suitable excipients by solvent casting method.

Results: Inclusion complexes prepared by ultrasonication method resulted in better solubility and dissolution rate compared to other methods studied. The fast dissolving films prepared using inclusion complexes were found to be transparent and smooth in texture. The thickness, drug content, $\%$ moisture absorption and moisture loss, folding endurance and weight variation was found to be uniform. In vitro release profiles were inversely proportional to the polymer and directly proportional to superdisintegrant and plasticizer concentration. Optimized formulations (F8 and F12) were subjected for fourier transform infrared spectroscopy (FT-IR) and differential scanning calorimetry (DSC) which revealed the absence of any chemical interactions between the excipients of the formulation. The formulations were found to be stable without any significant changes during the stability studies carried out as per the ICH guidelines.

Conclusion: The study revealed the successful development of fast dissolving films of lamotrigine with promising results thereby suggesting the possible use of the developed films in the rapid management of epileptic seizures.

Keywords: Fast dissolving films; epilepsy; lamotrigine; inclusion complex; HPMC; PVA; physico-chemical characterization.
\end{abstract}

\section{INTRODUCTION:}

Oral route of drug administration has wide acceptance and hence up to $50-60 \%$ of total dosage forms are administered orally. Solid dosage forms like tablets and capsules though popular but has certain limitations like difficulty in swallowing, risk of choking in the throat and always require some fluid to consume. This difficulty in swallowing or dysphasia is currently affecting $35 \%$ of general population ${ }^{1}$. Due to this problem, pediatric and geriatric patients, tend to avoid taking oral solid dosage forms like tablets and capsules due to fear of choking ${ }^{2}$.

Fast dissolving oral films (FDOFs) are the most advanced form of oral solid dosage forms due to more flexibility and comfort, improves the efficacy of drugs by dissolving within a minute in oral cavity after the contact with saliva without chewing and no need of water for FDOF's administration ${ }^{3}$. It gives quick absorption and instant bioavailability of drugs due to high blood flow and permeability. FDOFs are useful in patients such as pediatric, geriatrics, bedridden, emetic patients, diarrhea, sudden episode of allergic attacks, coughing or for those who have an active life style ${ }^{3}$.

Epilepsy is a neurological disorder which requires quick management of seizures in order to avoid the risk of permanent brain damage ${ }^{4}$. Thus, it is desirable to develop fast dissolving films for the anti-epileptic drugs. Lamotrigine is a new antiepileptic drug, which acts by @ 2011-14, JDDT. All Rights Reserved blocking voltage-dependent sodium channel, and reducing the release of excitatory neurotransmitters, particularly glutamate and aspartate. It is a bitter drug ${ }^{5}$ and also has poor aqueous solubility $\left(0.17 \mathrm{mg} \mathrm{ml}^{-1}\right.$ at $25^{\circ} \mathrm{C}$ ). Cyclodextrins have been used as increase the aqueous solubility of poorly water-soluble drugs and also to mask the bitter taste of drugs. Hence, in the present study, HP $\beta$ CD inclusion complexes were prepared to increase the solubility and to mask the bitter taste of lamotrigine. Further, these inclusion complexes were used to prepare the fast dissolving films with an aim the control the epileptic seizures in the shortest possible time.

\section{MATERIALS:}

Lamotrigine was received as a gift sample from Koppale Pharma Pvt Ltd, Hyderabad. HP B CD was received as a gift sample from Gangwal Chemicals Mumbai. HPMC, PVA, glycerol, citric acid, aspartame, crosspovidone were procured from SD Fine Chemicals, Mumbai.

*Corresponding Author: Dr. H Doddayya

Department of Pharmaceutics, N.E.T Pharmacy College, Navodaya Nagar, Raichur, Karnataka E-Mail ID: hdnet@ rediffmail.com Cell No: 9880043604 


\section{METHODS:}

\section{Phase solubility studies ${ }^{6}$ :}

Phase solubility studies were carried out in distilled water according to the method described by Highuchi and Connors. Constant amount of lamotrigine $(30 \mathrm{mg})$ that exceeded its solubility was added to $25 \mathrm{ml}$ of aqueous solutions of HP $\beta \mathrm{CD}$ in various molar concentrations $(2-20 \mathrm{mM})$. Then the suspensions were shaken on the rotary shaker at $25^{\circ} \mathrm{C}$ for 3 days. The samples were filtered, diluted and the concentration of lamotrigine was determined spectrophotometrically at $306 \mathrm{~nm}$. The apparent 1:1 stability constant was calculated from the phase solubility graph using the following equation,

$$
\mathrm{K}_{\mathrm{s}}=\frac{\text { Slope }}{\mathrm{S}_{0}(1-\text { Slope })}
$$

Where, $\mathrm{S}_{\mathrm{o}}$ is the solubility of lamotrigine in absence of HP $\beta$ CD.

Preparation of inclusion complexes ${ }^{7}$ : Lamotrigine and HP $\beta$ CD complexes were prepared by different methods using different molar concentrations of drug and HP $\beta$ $\mathrm{CD}(1: 1$ and $1: 2)$.

\section{Physical Mixture (PM):}

Lamotrigine and HP $\beta$ CD in the different molar ratios were mixed in a mortar for about 15 min with constant trituration, passed through sieve No. 80 and stored in a dessicator over fused calcium chloride

\section{Kneading Method (KN):}

Lamotrigine and HP $\beta$ CD in different molar ratios were triturated by adding small quantity of $50 \%$ ethanol to get slurry like consistency, continued stirring for $1 \mathrm{~h}$. Slurry was further dried at $25^{\circ} \mathrm{C}$ troom temperature for $24 \mathrm{~h}$.
Pulverized and passed through sieve No. 80 and stored in a dessicator over fused calcium chloride.

\section{Ultrasonication Method:}

Lamotrigine and HP $\beta$ CD in different molar ratios were triturated by adding small quantity of 50\% ethanol to get slurry like consistency and kept in ultrasonicator for $1 \mathrm{hr}$. Slurry was further dried at $25^{\circ} \mathrm{C} t$ room temperature for 24 h. Pulverized and passed through sieve No. 80 and stored in a dessicator over fused calcium chloride.

The prepared inclusion complexes were subjected for FT-IR and DSC studies. Dissolution studies for the inclusion complexes were also carried out using $900 \mathrm{ml}$ of $0.1 \mathrm{~N} \mathrm{HCl}$ as dissolution medium at $50 \mathrm{rpm}$ and 37.0 $\pm 0.5^{\circ} \mathrm{C}$.

\section{Preparation of fast dissolving films:}

The fast dissolving films of lamotrigine were prepared by solvent casting method $^{8}$ using the prepared inclusion complexes with different concentrations of polymers like HPMC and PVA, glycerin as plasticizer, citric acid as saliva stimulant, aspartame and crosspovidone were used as sweetening agent and superdisintegrant respectively. Formulation codes and their respective compositions are given in the Table: 1 . The inclusion complex equivalent to $5 \mathrm{mg}$ drug was dissolved in $10 \mathrm{ml}$ of distilled water and required quantities of the above excipients were added and stirred on magnetic stirrer for $1 \mathrm{hr}$. The solution was degassed to remove entrapped air by using ultrasonicator. The prepared solution was casted on mercury employing circular shaped ring of $13.195 \mathrm{~cm}^{2}$ and air dried for $24 \mathrm{~h}$. The films were carefully removed and cut into required size $\left(2 \times 2 \mathrm{~cm}^{2}\right)$ to deliver the dose equivalent to $5 \mathrm{mg}$ per strip. The films were stored in aluminium foil and placed in airtight containers for further studies.

Table: 1 Formulation of fast dissolving film of lamotrigine

\begin{tabular}{|c|c|c|c|c|c|c|c|c|c|c|c|c|c|c|}
\hline Code & F1 & F2 & F3 & F4 & F5 & F6 & F7 & F8 & F9 & F10 & F11 & F12 & F13 & F14 \\
\hline HPMC & 200 & 250 & 300 & 200 & 200 & 200 & 200 & 200 & 200 & 200 & 200 & 50 & 100 & 150 \\
\hline PVA & - & - & - & - & - & - & - & - & - & - & - & 150 & 100 & 50 \\
\hline Glycerin & 0.1 & 0.1 & 0.1 & 0.2 & 0.3 & 0.4 & 0.2 & 0.2 & 0.2 & 0.2 & 0.2 & 0.2 & 0.2 & 0.2 \\
\hline CP & - & - & - & - & - & - & 2 & 4 & 6 & - & 4 & 4 & 4 & 4 \\
\hline
\end{tabular}

Citric acid (100mg) Aspartame (5mg) and Pineapple flavor $(0.1 \mathrm{ml})$ are constant in all the formulations

\section{Evaluation of fast dissolving films:}

\section{Weight variation test ${ }^{9}$ :}

This test was carried out by taking $2 \times 2 \mathrm{~cm}^{2}$ of the film cut at three different places from the casted film. The weight of each film was taken individually using electronic balance. Average of three readings was taken for weight variation study.

\section{Thickness of Film ${ }^{10}$ :}

All the batches were evaluated for thickness by using calibrated Vernier caliper. Three readings from all the batches were taken and mean thickness was evaluated.

Drug content uniformity ${ }^{11}$ :
Drug content of all films was determined by UVSpectrophotometric method. For this $2 \times 2 \mathrm{~cm}^{2}$ film was cut and dissolved in $100 \mathrm{ml}$ of $0.1 \mathrm{~N} \mathrm{HCl}$. The solution was filtered and absorbance was recorded at $267.0 \mathrm{~nm}$ using UV-Spectrophotometer. Drug content was calculated using the calibration curve constructed in the same media. Triplicate reading were taken and the average value was considered as drug content of the films.

\section{Percentage moisture absorption ${ }^{12}$ :}

Films were cut into $2 \times 2 \mathrm{~cm}^{2}\left(4 \mathrm{~cm}^{2}\right)$. The moisture uptake by the films $(\mathrm{n}=3)$ was determined by exposing them to an environment of $75 \%$ relative humidity (saturated solution of potassium sulphate) at room temperature for 1 day. The uptake of moisture by the 
films was measured and percentage moisture absorption was calculated by using the following formulae:

$$
\% \text { moisture absorption }=\frac{\text { Final weight }- \text { initial weight }}{-------------- \text { x100 }}
$$

Percentage moisture loss ${ }^{13}$ :

Percentage moisture loss was calculated to check the integrity of films at dry condition. Three films $2 \times 2$ $\mathrm{cm}^{2}$ was cut out and weighed accurately and kept in desiccators containing fused anhydrous calcium chloride. After 72 hours the films were removed and reweighed. The percentage moisture loss was calculated using the formula:

$$
\% \text { moisture absorption }=\frac{\text { Final weight }- \text { initial weight }}{-- \text { Initial weight }^{-14}}
$$

\section{Folding endurance ${ }^{14}$ :}

The folding endurance is related to the flexibility of a film and it was measured manually by firmly folding the films repeatedly through the middle. The number of folds on the same crease, required to produce crack in the film was noted as the value of folding endurance.

\section{Surface $\mathrm{pH}$ determination ${ }^{14}$ :}

As an acidic or alkaline $\mathrm{pH}$ may cause irritation to the oral mucosa, it was determined to keep the surface $\mathrm{pH}$ as close to neutral as possible. A combined $\mathrm{pH}$ electrode was used for this purpose. Oral films were slightly wet with the help of water. The $\mathrm{pH}$ was measured by bringing the electrode in contact with the surface of the oral film. The experiments were performed in triplicate, and average values were reported.

\section{Fourier transformer infrared spectroscopy (FTIR) study:}

The compatibility between drug and polymer was detected by IR spectra obtained on Shimadzu 8400 instrument, Japan. The pellets were prepared on $\mathrm{KBr}$ press (spectra lab, India). The spectra were recorded over the wave number range of 4000 to $400 \mathrm{~cm}^{-1}$.

\section{Differential scanning calorimeter (DSC) study:}

Thermograms were obtained by using a differential scanning calorimeter (DSC Q20 V24.4 Build 116, Japan.) at a heating rate of $10^{\circ} \mathrm{C} / \mathrm{min}$ over a temperature range of $0-200^{\circ} \mathrm{C}$ The sample was hermetically sealed in an aluminum crucible. Nitrogen gas was purged at the rate of $10 \mathrm{ml} / \mathrm{min}$ for maintaining inert atmosphere.

\section{In vitro disintegration time:}

Disintegration test was performed in the USP disintegration apparatus. The films were placed in the tubes in the container and the disks were placed over it. The average disintegration time from each formulation was noted in triplicate.

\section{In vitro dissolution studies of films:}

The prepared fast dissolving films were subjected to in vitro dissolution studies using an 8 station USP (TypeII) dissolution apparatus (Electro Lab, TDT-O8L,
Mumbai). The dissolution studies were carried out in $900 \mathrm{ml}$ of $0.1 \mathrm{~N} \mathrm{HCl}$ at $37 \pm 0.5^{\circ} \mathrm{C}$. The speed of the paddle was set at $50 \mathrm{rpm}$. Sampling was done every 1 min interval up to 10 minutes, there after 5 minutes interval up to $30 \mathrm{~min}$ and finally at 45,60 and $90 \mathrm{~min}$ respectively. For each sample, $5 \mathrm{ml}$ of sample was withdrawn from the dissolution medium and replaced with equal volume of fresh medium. The samples withdrawn were analyzed in the UV spectrophotometer at $267 \mathrm{~nm}$.

\section{Stability studies ${ }^{14}$ :}

A stability study of 1 month was carried out for optimized batches at $40^{\circ} \mathrm{C} \pm 2{ }^{\circ} \mathrm{C}$ and $75 \% \pm 5 \%$ relative humidity, $30^{\circ} \mathrm{C} \pm 2{ }^{\circ} \mathrm{C}$ and $75 \% \pm 5 \%$ relative humidity. After every one month the films were evaluated for physical appearance, weight variation, drug content, disintegration time and dissolution time.

\section{RESULTS AND DISCUSSION:}

\section{Phase solubility study of lamotrigine with HP $\beta$ CD:}

The phase solubility graph for the complex formation between lamotrigine and HP $\beta$ CD is shown in Fig. 1. Drug solubility increased with increase in the concentration of HP $\beta \mathrm{CD}$. The slope calculated was 0.284 which is less than 1 , thus $1: 1$ stoichiometry was suggested. The value of the stability constant was found to be $470 \mathrm{M}^{-1}$ with high regression value of 0.936 indicating the formation of instantaneous and high order inclusion complexes between the lamotrigine and HP $\beta$ $\mathrm{CD}^{15}$ and 16 .

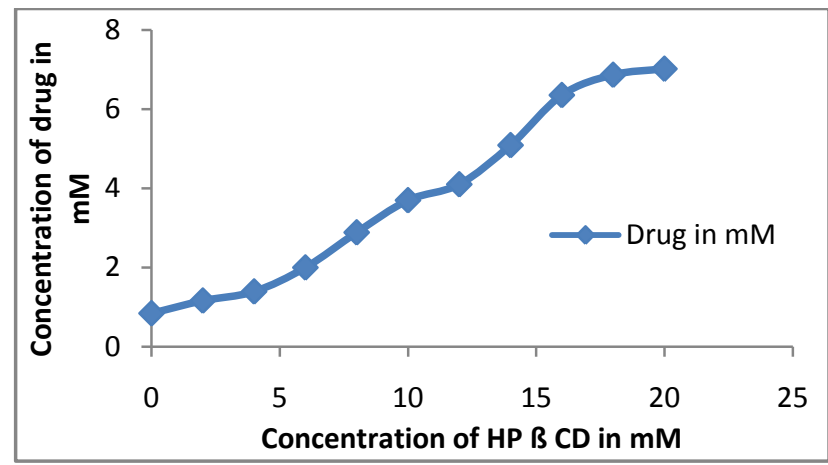

Fig: 1 Phase solubility diagram of lamotrigine with HP $\beta$ CD

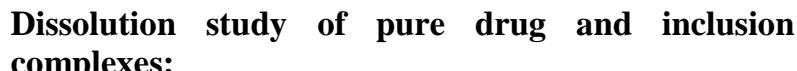
complexes:

In vitro release studies of pure drug and inclusion complexes (1:1 and 1:2) were performed using $0.1 \mathrm{~N}$ $\mathrm{HCl}$. The study revealed a significant increase in dissolution rate of lamotrigine from the inclusion complexes compared to its pure form. Among the three different methods, inclusion complexes prepared by ultrasonication method exhibited higher rate of dissolution compared to the kneading, physical mixture and pure drug. Improved dissolution observed in case of ultrasonication method may be due to the formation of solid inclusion complexes, with better interaction of drug and HP $\beta$ CD during the ultrasonicating process. Hence inclusion complexes prepared by ultrasonication method (1:1 molar ratio of drug and HP $\beta$ CD) were 


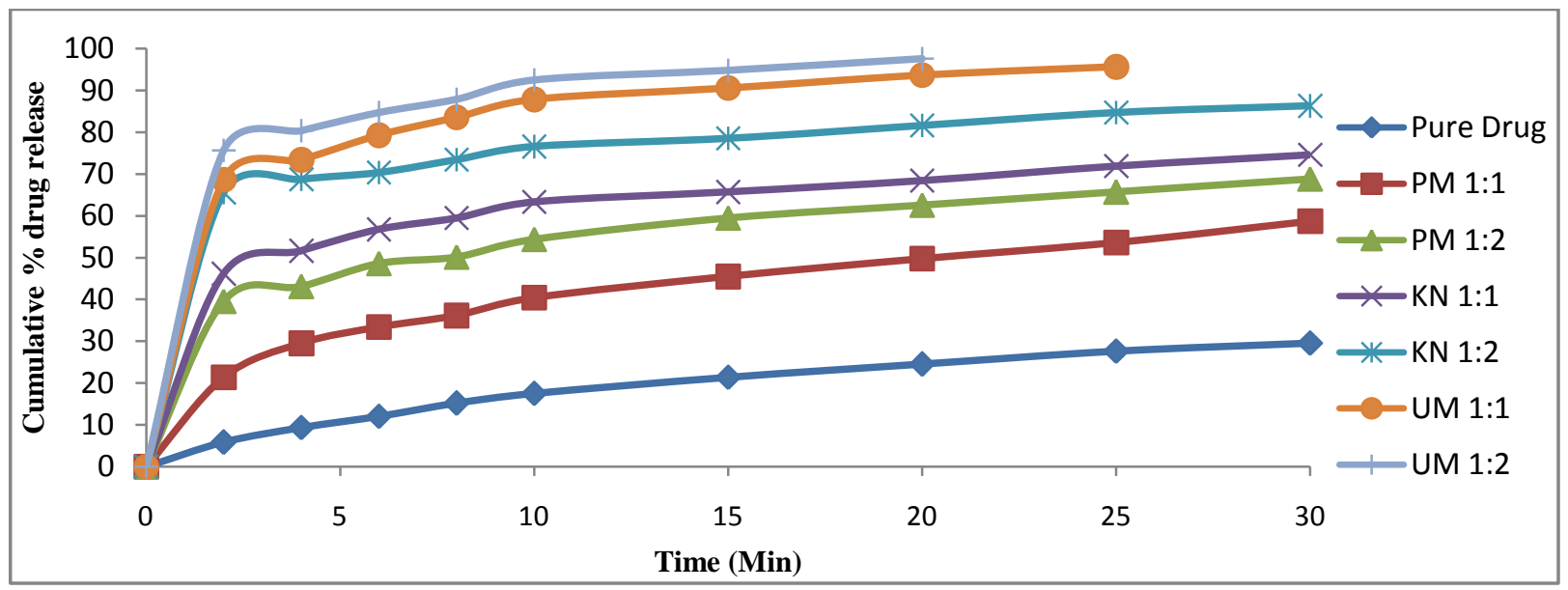

Fig: 2 In vitro dissolution of pure drug an d inclusion complexes

Physicochemical characterization of fast dissolving films:

\section{Weight, thickness and drug content of the films:}

The weight of the fast dissolving films were found to be uniform from batch to batch and were found to be between $59.07 \pm 0.05$ to $67.59 \pm 0.1 \mathrm{mg}$ and the thickness was varied between $0.154 \mathrm{~mm} \pm 0.001$ to $0.193 \mathrm{~mm} \pm 0.001$. The results showed that weight and thickness of the films proportionately increased with increase in polymer concentration. The low standard deviation values ensured the weight and thickness uniformity of the prepared films. The drug content in all the formulations was found to be between $98.27 \pm 0.041$ to $99.70 \pm 0.144 \%$. The results showed that, the drug content was uniform and reproducible in each batch of different fast dissolving film formulations.

Table: 2 Physicochemical evaluation of fast dissolving films of lamotrigine*

\begin{tabular}{|c|c|c|c|c|c|c|c|c|}
\hline Code & $\begin{array}{c}\text { Average } \\
\text { Weight } \\
(\mathrm{mg})\end{array}$ & $\begin{array}{l}\text { Thickness } \\
(\mathrm{mm})\end{array}$ & $\begin{array}{c}\text { Drug content } \\
(\%)\end{array}$ & $\begin{array}{c}\text { Moisture } \\
\text { absorption } \\
(\%)\end{array}$ & $\begin{array}{l}\text { Moisture } \\
\text { loss }(\%)\end{array}$ & $\begin{array}{c}\text { Folding } \\
\text { endurance }\end{array}$ & Surface $\mathrm{pH}$ & $\begin{array}{c}\text { Disintegration } \\
\text { time }(\mathrm{sec})\end{array}$ \\
\hline F1 & $59.55 \pm 0.2$ & $0.157 \pm 0.002$ & $99.41 \pm 0.061$ & $8.71 \pm 0.25$ & $10.47 \pm 0.49$ & $322.33 \pm 2.51$ & $6.66 \pm 0.15$ & $24 \pm 0.57$ \\
\hline $\mathrm{F} 2$ & $62.43 \pm 0.11$ & $0.165 \pm 0.002$ & $98.95 \pm 0.328$ & $11.6 \pm 0.36$ & $8.37 \pm 0.33$ & $284.66 \pm 1.52$ & $6.33 \pm 0.11$ & $26 \pm 0.57$ \\
\hline F3 & $64.12 \pm 0.05$ & $0.179 \pm 0.001$ & $98.93 \pm 0.371$ & $13.83 \pm 0.76$ & $6.45 \pm 0.46$ & $257.33 \pm 3.05$ & $6.63 \pm 0.057$ & $29 \pm 1.52$ \\
\hline $\mathrm{F} 4$ & $60.03 \pm 0.10$ & $0.157 \pm 0.001$ & $98.76 \pm 0.353$ & $9.56 \pm 0.44$ & $9.36 \pm 0.42$ & $327.33 \pm 1.15$ & $6.76 \pm 0.152$ & $23 \pm 0.57$ \\
\hline F5 & $60.37 \pm 0.21$ & $0.158 \pm 0.001$ & $98.70 \pm 0.156$ & $10.83 \pm 0.75$ & $8.42 \pm 0.37$ & $333.66 \pm 2.08$ & $6.46 \pm 0.152$ & $20 \pm 0.57$ \\
\hline F6 & $61.03 \pm 0.05$ & $0.157 \pm 0.002$ & $99.13 \pm 0.045$ & $12.68 \pm 0.27$ & $7.19 \pm 0.43$ & $342.33 \pm 3.05$ & $6.63 \pm 0.208$ & $18 \pm 0.57$ \\
\hline F7 & $65.09 \pm 0.07$ & $0.171 \pm 0.001$ & $98.82 \pm 0.122$ & $11.65 \pm 0.33$ & $8.52 \pm 0.28$ & $315.33 \pm 3.05$ & $6.66 \pm 0.208$ & $16 \pm 0.57$ \\
\hline F8 & $66.42 \pm 0.20$ & $0.183 \pm 0.002$ & $99.44 \pm 0.120$ & $13.39 \pm 0.12$ & $8.06 \pm 0.06$ & $305.66 \pm 2.51$ & $6.83 \pm 0.057$ & $13 \pm 1$ \\
\hline F9 & $67.59 \pm 0.1$ & $0.193 \pm 0.001$ & $98.27 \pm 0.041$ & $15.60 \pm 0.35$ & $7.57 \pm 0.26$ & $295.33 \pm 3.05$ & $6.53 \pm 0.208$ & $17 \pm 2$ \\
\hline F10 & $59.07 \pm 0.05$ & $0.154 \pm 0.001$ & ----- & $9.83 \pm 0.76$ & $6.85 \pm 0.08$ & $325.66 \pm 1.15$ & $6.66 \pm 0.251$ & $32 \pm 1.15$ \\
\hline F11 & $65.86 \pm 0.09$ & $0.183 \pm 0.002$ & ----- & $12.68 \pm 0.42$ & $8.50 \pm 0.16$ & $303.33 \pm 2.08$ & $6.5 \pm 0.3$ & $18 \pm 3.05$ \\
\hline F12 & $61.20 \pm 0.04$ & $0.181 \pm 0.001$ & $99.25 \pm 0.092$ & $8.76 \pm 0.40$ & $8.53 \pm 0.19$ & $311.66 \pm 1.52$ & $6.73 \pm 0.057$ & $14 \pm 2$ \\
\hline F13 & $62.46 \pm 0.07$ & $0.182 \pm 0.003$ & $98.83 \pm 0.087$ & $10.37 \pm 0.54$ & $8.29 \pm 0.06$ & $315.33 \pm 0.57$ & $6.43 \pm 0.152$ & $16 \pm 1.52$ \\
\hline F14 & $63.15 \pm 0.06$ & $0.183 \pm 0.001$ & $99.70 \pm 0.144$ & $12.59 \pm 0.36$ & $8.22 \pm 0.03$ & $321.33 \pm 2.08$ & $6.76 \pm 0.057$ & $18 \pm 1.15$ \\
\hline
\end{tabular}




\section{Percentage moisture absorption and loss:}

The percentage moisture absorption test was carried out to check physical stability or integrity of the film at humid condition. Among all, the formulations containing higher concentration of HPMC and glycerin showed greater moisture absorption compared to the formulations containing lower concentration of HPMC. Glycerin and HPMC both being hydrophilic in nature has the tendency to increase the moisture absorption ${ }^{17}$. Fast dissolving films containing the PVA along with HPMC showed more moisture absorption compared to those containing HPMC alone. This could be probable be due more hydrophilic nature of PVA than HPMC.

The percentage moisture loss study was carried out to check the integrity of the fast dissolving films at dry condition. Percentage moisture loss was inversely proportional to the HPMC concentration in the films. Also, as the glycerin concentration was reduced, \% moisture loss was also decreased. It is obvious to note that, these hydrophilic excipients tend to hold the moisture and their reduced levels in the films may lead to higher moisture loss.

\section{Folding endurance:}

The folding endurance of a film is frequently used to estimate the ability of the film to withstand repeated bending, folding and creasing and may be encountered as a measure of the quality of the films. The folding endurance of different fast dissolving films was in the range of $257.33 \pm 3.05$ to $342.33 \pm 3.05$. Folding endurance of HPMC films increased with increase in concentration of HPMC and glycerin. This could be due to more elasticity of polymer at higher levels of HPMC in the films and also as the plasticizer concentration increases, flexibility of the film also increases and thus the folding endurance too ${ }^{18}$.

\section{Surface pH determination:}

The prepared films $\mathrm{pH}$ should be in the neutral range to prevent the irritation and damage to the oral mucosa. The surface $\mathrm{pH}$ of formulated fast dissolving films was found to be in the range of 6.33 to 6.83 which indicates that, the formulated fast dissolving films were in the neutral $\mathrm{pH}$ range and would not cause any irritation after placing in the oral cavity.

\section{FT-IR \& DSC studies:}

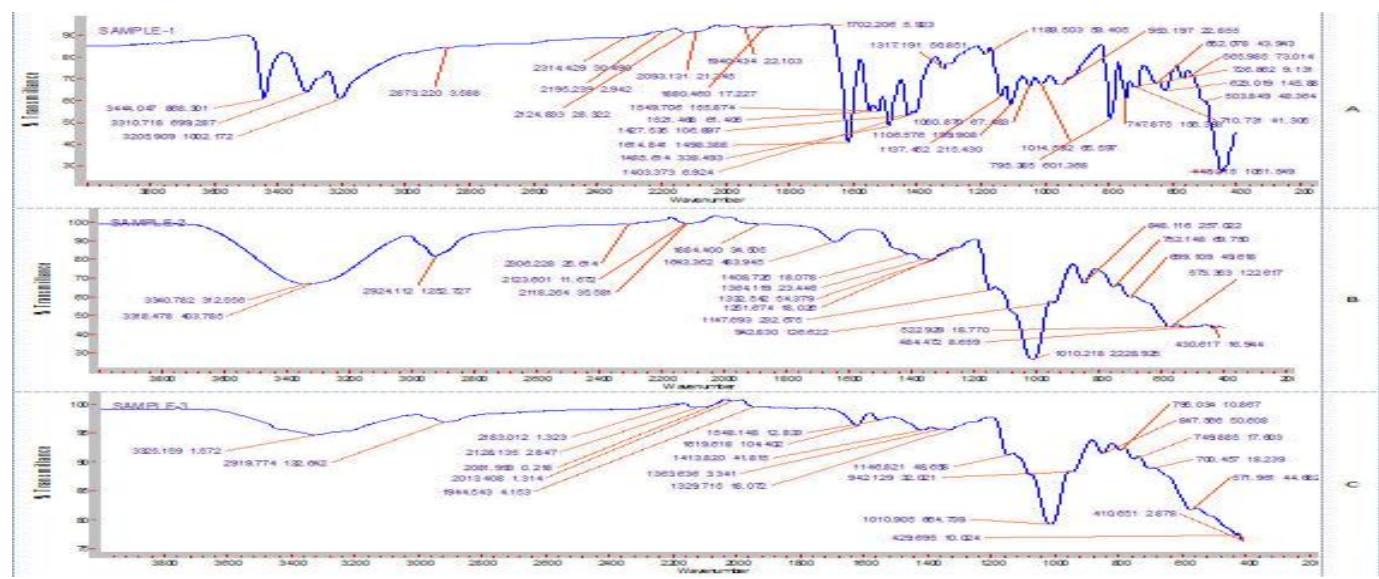

Fig: 4 FT-IR spectra of [A] Pure drug lamotrigine, [B] Pure HP B CD and [C] Inclusion complexes.

The IR spectrum of the drug lamotrigine exhibited its characteristic absorption bands at 3311 \& 3206 for N-H stretching of $\mathrm{NH}_{2}$ groups, 3020 for Aromatic $\mathrm{C}-\mathrm{H}$ stretching, 1615 for $\mathrm{C}=\mathrm{N}$ stretching, 1550, 1521 and 1485 for $\mathrm{C}=\mathrm{C}$ ring stretching, $1412 \& 1317$ for $\mathrm{C}-\mathrm{N}$ stretching, 795 for tri substituted phenyl ring and 747 for $\mathrm{C}-\mathrm{Cl}$.

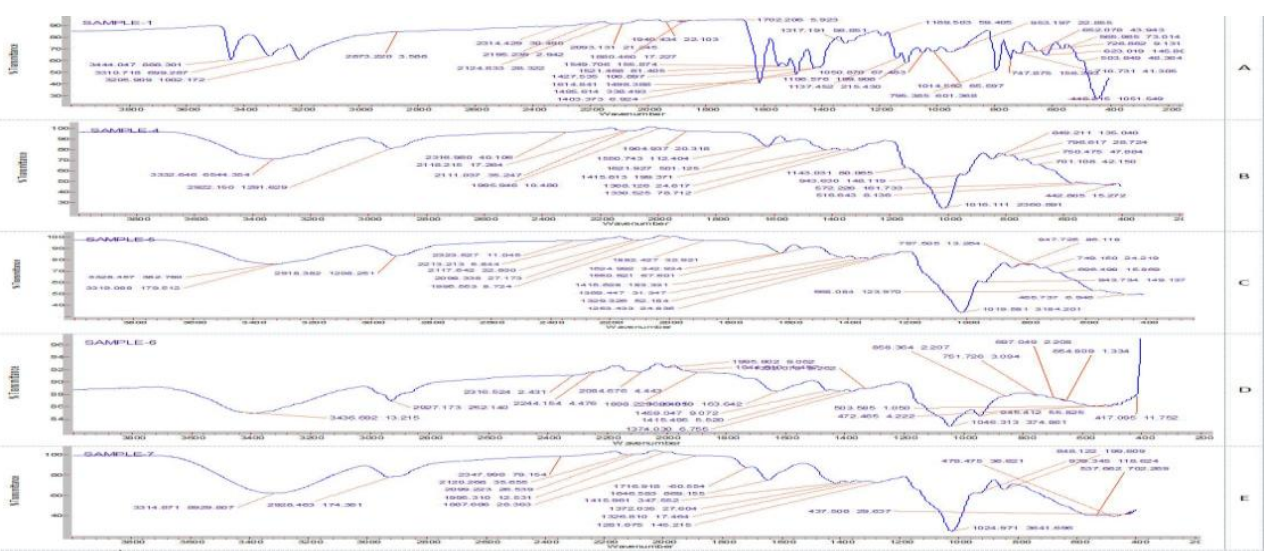

Fig: 5 FT-IR specta of [A] Pure drug lamotrigine, [B] physical mixture of inclusion complex with HPMC, [C] physical mixture of inclusion complex with HPMC and PVA, [D] Optimized formulation (F8) and [E] Optimized formulation (F12) 
The IR spectra of the physical mixture of drug and HP $B$ $\mathrm{CD}$ revealed no significant changes in the absorption bands compared to that of pure drug. However, the IR of the optimized inclusion complex showed certain changes in the absorption bands. It can be interpreted that the functional group like $\mathrm{NH}_{2}$ on the ring of the drug may interact with $\mathrm{OH}$ group of the cyclodextrin through hydrogen bonding resulting for the changes in the positions of characteristic bands in the spectrum of inclusion complex of drug with the cyclodextrin. The IR spectra of physical mixtures did not show any significant changes in the positions of absorption bands when compared to pure drug indicating the compatibility of the drug with the excipients used. However, some minor and insignificant changes were observed in the spectral bands in the IR spectra of the optimized formulations and the major peaks remained intact suggesting the integrity of the drug. Hence it can be concluded that the interaction is due to hydrogen bonding between drug and cyclodextrin in the inclusion complexes and no chemical interaction in the formulations prepared from inclusion complex.

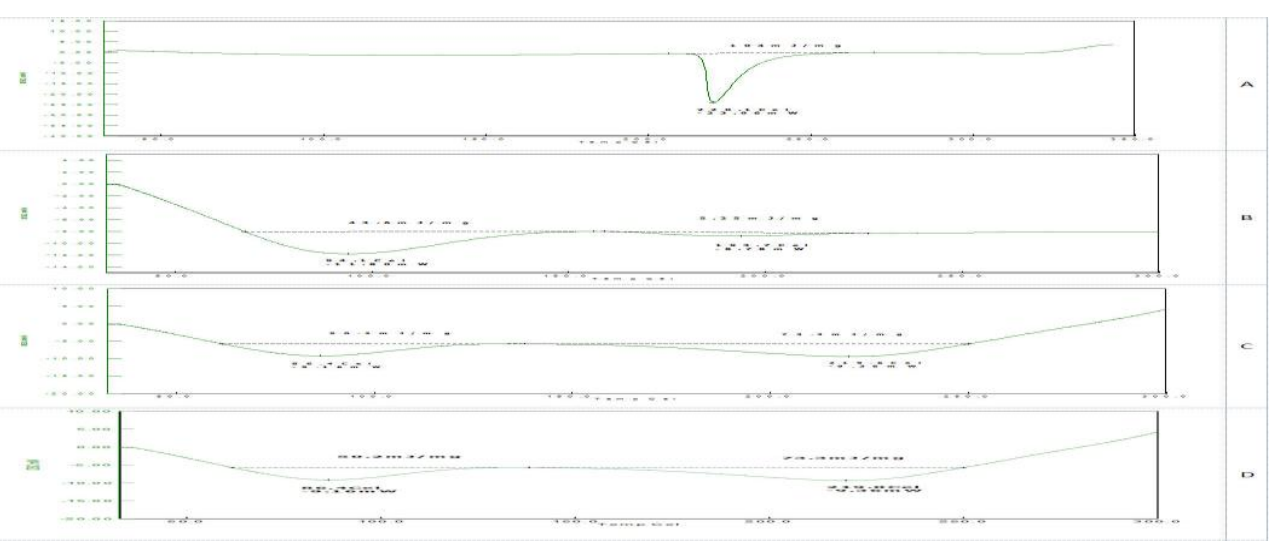

Fig: 6 DSC thermograms of [A] Pure drug lamotrigine, [B] Inclusion complexes, [C] Optimized formulation (F8) and $[D]$ optimized formulation (F12).

The DSC thermogram of pure drug lamotrigine showed an intense and significant endothermic peak at temperature of $220^{\circ} \mathrm{c}$ which corresponds to the point of the drug $\left(214-220^{\circ} \mathrm{c}\right)$ reported in the literature ${ }^{19}$, thus confirming the purity and identity of the drug.

As observed in Fig: 6(B), the sharp endothermic peak of the drug is changed to a very broad endothermic peak in the thermogram of drug which is possible only when the drug undergoes certain changes like interaction with the HP $\beta C D$ resulting in the formation of inclusion complex. The DSC thermograms of formulation containing inclusion complexes with HPMC and formulation containing inclusion complexes with HPMC and PVA do not show any appreciable significant change in the melting point range and nature of the peaks as compared with the pure drug and the inclusion complex. Overall, the FT-IR and DSC studies conclusively proved the integrity of the drug in the pure form and also in its different formulations.

\section{Disintegration time:}

The disintegration times of the films were in the range of $13 \pm 1.0$ to $32 \pm 1.15$ which was largely dependent on the various formulation variables. The disintegration time was proportionately increased with increase in polymer levels in the films, whereas it reduced with increase in plasticizer concentration which could be probably due to tendency of glycerin to increase the wettability of the films. The disintegration time was proportionately reduced with increase in superdisintegratant concentration only at certain level. Increase in superdisintegrant concentration from 4 to $6 \%$ could not significantly reduce the disintegration time, which could be due to blockade of capillary pores preventing the entry of fluid in to the films ${ }^{20}$. The presence of cyclodextrin complex also reduced the disintegration time of the films compared to those which are prepared in absence of the complexes. This could be due to hydrophilic nature of the cyclodextrins which resulted in reduced disintegration time. Compared to the films prepared using HPMC alone, the combination of HPMC and PVA films showed lesser disintegration time. PVA being more hydrophilic, its presence in the HPMC films resulted in reduced disintegration time ${ }^{21}$.

\section{In vitro dissolution:}

The in vitro disintegration and dissolution are the key factors that determine the successful development of fast dissolving films. The results of the dissolution study revealed that as the polymer concentration in the films increased there was a proportionate decrease in drug release. This could be due to the extensive swelling of the polymer and formation of thick gel structure that eventually decreased drug release from the films. It was observed that as the plasticizer concentration in the films increased there was a proportionate increase in the drug release. Glycerin being a hydrophilic excipient and a good solubilizer might have increased the drug release. Though, the films prepared using higher levels of plasticizer though showed better dissolution rate but resulted in stickiness of the films. Hence, plasticizer concentration of $1 \% \mathrm{v} / \mathrm{v}$ was kept constant. Increase in crosspovidone concentration from 2 to $4 \%$ has significantly increased the drug release from the fast dissolving films. However, further increase up to $6 \%$ 
could not significantly increase the drug release. This could be probably due to blockade of capillary pores at the higher concentration of crosspovidone. The absence of inclusion complex (F10) resulted in much slower drug release that extended up to $95.62 \%$ at the end of $60 \mathrm{~min}$ dissolution study. However, little improvement in dissolution rate was observed in case of formulation F11 (99.52\% in $30 \mathrm{~min}$ ), which was prepared with pure drug but in combination with $4 \%$ crosspovidone. Compared to the fast dissolving films containing inclusion complexes both the above formulations failed to release the drug in the shortest possible time. The study revealed that, presence of inclusion complexes can significantly increase the drug release from the fast dissolving films.
During the optimization process, films were prepared by the combination of HPMC and PVA in different ratios (F12 to F14) and their effect on dissolution rate was studied. The films containing higher levels of PVA than HPMC (F12) resulted in faster dissolution rate $(98.77 \%$ in $6 \mathrm{~min}$ ) compared to those containing equal (F13, $99.57 \%$ in $8 \mathrm{~min}$ ) and higher (F14, 96.38\% in $8 \mathrm{~min}$ ) amount of HPMC. This could be possibly due to more hydrophilic nature of PVA that resulted in improved dissolution of the films. In conclusion, the results of dissolution study revealed that, formulation variables play in important role in the achievement of rapid dissolution of the drug from the fast dissolving films.

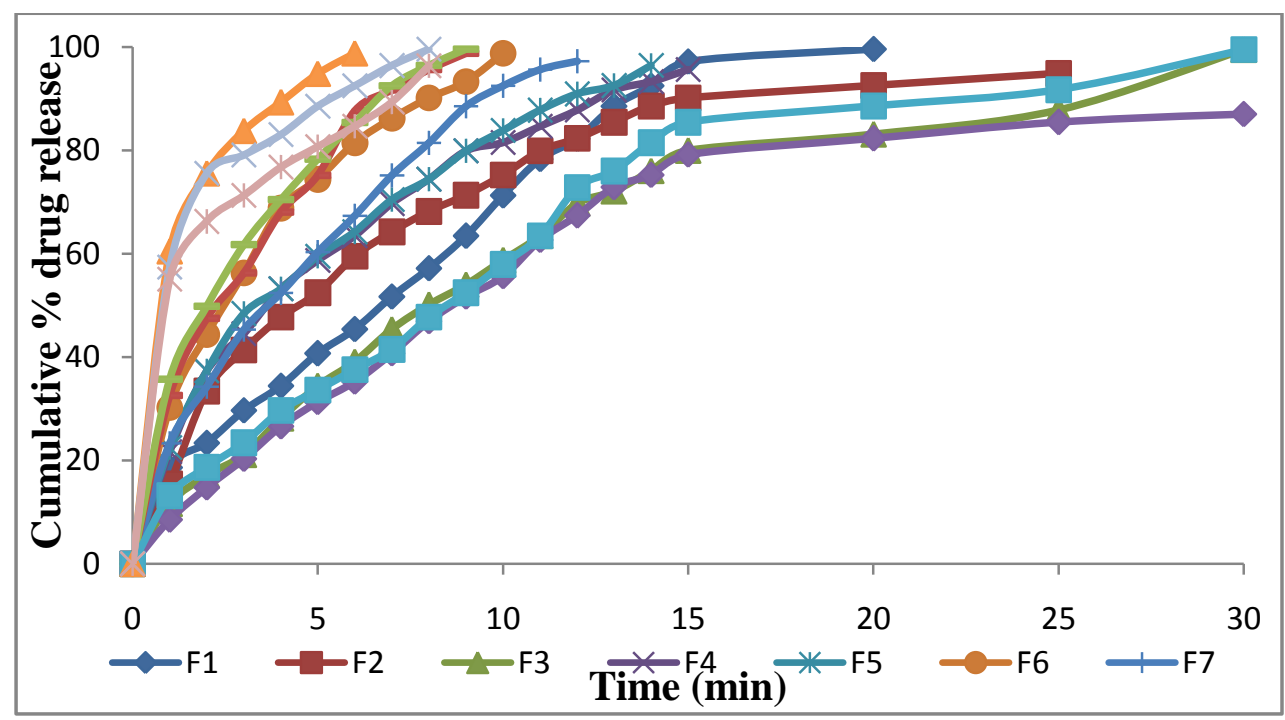

Fig: 7 In vitro dissolution profile of fast dissolving films of lamotrigine

\section{Stability studies:}

The stability studies were carried out for the optimized formulations ( $\mathrm{F} 8$ and $\mathrm{F} 12$ ) at $40 \pm 2^{\circ} \mathrm{C} / 75 \pm \% 5 \mathrm{RH}$ as per $\mathrm{ICH}$ guidelines for a period of one month and evaluated for their physical appearance, weight variation, drug content and drug release study. No significant changes in the appearance, weight of the fast dissolving films was observed during the stability study. The drug content of F8 and F12 after stability studies was found $98.69 \pm 0.19$, and $99.08 \pm 0.18 \%$ respectively. There was no significant variation in $\mathrm{T}_{50 \%}$ and $\mathrm{T}_{90 \%}$ at the end of one month stability period. This indicated that the fast dissolving films remained fairly stable at $40^{\circ} \pm 2 \mathrm{C}, 75 \% \mathrm{RH}$.

\section{CONCLUSION:}

In the present study fast dissolving films of lamotrigine were prepared by the incorporation of inclusion complexes and the film forming agents like HPMC, PVA and other excipients by solvent casting method. The prepared films exhibited uniform thickness, weight, drug content and rapid disintegration. The incorporation of HP ß CD inclusion complexes and superdisintegrants greatly improved the drug release from the prepared films. Over all the fast dissolving films of lamotrigine were successfully prepared and the obtained in vitro results were promising for the effective control of epileptic seizures in the shortest possible time.

CONFLICT OF INTEREST: We the authors, do not have any personal or financial conflict regarding the publication of this manuscript.

\section{ACKNOWLEDGEMENTS:}

The authors are thankful to M/S Kopalle Pharama Pvt.Ltd.Hyderabad and M/S Gangwal Chemicals Mumbai for providing pure drug lamotrigine and HP B $\mathrm{CD}$ respectively. The authors are also thankful to the management of Navodaya Educational Trust, Raichur for providing necessary research facilities to carry out the present work. 


\section{REFERENCES:}

1. Mohire NC, Yadav AV, “ Novel approach to formulate $\beta$ cyclodextrin complexed mouth dissolving tablet of metronidazole and its in-vitro evaluation" Journal of Pharmaceutical Research, 2010, 3(3), 662-667.

2. Mandeep k, Rana A.C, Nimrata S, "Fast Dissolving Films An Innovat Drug Delivery System" International Journal of Pharmaceutical Research and Allied Sciences, 2013, 2(1), 1424.

3. Deepak H, Aggarwal G and. Hari Kumar SL, “ Recent trends of fast dissolving drug delivery system" An International Research Journal of Pharmacophore, 2013, 4 (1), 1-9.

4. Patil RR, Shende AJ, Devarajan PV, "Microemulsion of lamotrigine for nasal delivery" Indian Journal of Pharmaceutical Sciences, 2007, 69(5), 721-722.

5. Amrutkar PP, Patil SB, Todarwal AN, Wagh MA, Kothawade PD, Surawase RK "Design and evaluation of taste masked chewable dispersible tablet of lamotrigine by melt granulation" International Journal of Drug Delivery, 2010, 2, 183-191.

6. Goudanavar P, Shah SH, Hiremath D, "Developement and characterization of Lamotrigine orodispersible tablets: inclusion complex with hydroxypropyl B cyclodextrin" International Journal of Pharmacy and Pharmaceutical Sciences, 2011, 3(3), 208-214.

7. Patil SS, Doddayya H, Gupta VRM, Gupta KS, "Effect of pH, selected cyclodextrins and complexation methods on solubility of lornoxicam" International Journal of Pharmacy and pharmaceutical Sciences, 2014 , 6(8) :,324-327.

8. Arya A, Chandra A, Sharma V, Pathak K, "Fast Dissolving Oral Films: An Innovative Drug Delivery System and Dosage Form" International Journal of Chemical Technology and Research, 2010, 2(1), 576-583.

9. Bhupinder B, Sarita $J$ "Formulation and evaluation of fast dissolving sublingual films of Rizatriptan Benzoate" International Journal of Drug Development and Research, 2012, 4(1), 133-143

10. Shelke PV, Dumbare AS, Godhave MV, Jadhav SL, Sonawane AA, Gaikwad DD, "Formulation and evaluation of rapidly disintegrating film of amlodipine besylate" International Journal of drug delivery \& therapeutics, 2012 , 2(2) , 72-75

11. Sabar MH. "Formulation and in vitro evaluation of fast dissolving film containing amlodipine besylate solid dispersion" International Journal of Pharmacy and Pharmaceutical Sciences, 2013, 5(4), 419-428.

12. EL- Nabarawi MA, Makky AM, EL-Setouhy DA, ABD Elmonem RA, Jasti BA "Development and characterization of orobuccal film". International Journal of Pharmacy and Pharmaceutical Sciences, 2012, 4(4), 186-193.

13. Nagar M, Nagar M, Chopra V, "Formulation and evaluation of mouth dissolving film of antipsychotic drug aripiprazole" Der pharmacia letter, 2012 , 4(4) ,1221-1227.

14. Jani M, Pandya H, "Formulation and evaluation of fast releasing film of odansetron hydrochloride" International Journal of Pharmaceutical Sciences 2012, 3(4), 2463-2476.

15. Bhise SD. Effect of Hydroxypropyl $\beta$-Cyclodextrin Inclusion Complexation on Solubility of Fenofibrate. International Journal of Research in Pharmaceutical and Biomedical Sciences, 2011, 2 (2), 596-604.

16. Ghodke DS, chaulang GM, Patil KS, Nakhat PD, Yeole P G, Naikwade NS, Magdum CS, "Solid State Characterization of Domperidone: Hydroxypropyl- $\beta$-Cyclodextrin Inclusion Complex" Indian Journal of Pharmaceutical Sciences, 2010, 72(2), 245-249.

17. Nappinnai M, Chandanbala R, Balaijirajan R, "Formulation and evaluation of nitrendipine buccal films" Indian Journal of Pharmaceutical Sciences, 2008, 70(5), 631-635.

18. Patil P, Shrivastava SK, "Formulation, evaluation and optimization of fast dissolving oral film of selective antihypertensive drug" World Journal of pharmacy and pharmaceutical Sciences, 2014, 3(8), 996-1060.

19. Gangavarapu PK, Dr. Prasad GS, "Physicochemical Properties of Lamotrigine and its Compatibility with Various Inactive Ingredients to Formulate Lamotrigine Orally Disintegrating Tablets" International Journal of Pharmaceutical and Biological Archives, 2013, 4(1), 94-95.

20. Patil A, Ghorwade V, Patil S, Ikkurthi K, Inuganti KS, Porandla V, "Formulation and evaluation of monteluksat sodium fast dissolving films by using gelatin as a film base" Research Journal of pharmaceutical, Biological and Chemical. Sciences, 2011, 2(3), 880-888.

21. Chauhan NS, Tomar A, Sharma K, Mittal A, Bajaj U, "Formulation and evaluation of fast dissolving oral film of dicyclomine as potential route of buccal delivery" International.Journal of drug development and Research, 2012, 4(2), 408-417 